\title{
COMBINATION OF AM AND FM SCREENING AS SECURITY PRINTED FEATURE
}

\author{
Vladislav Vereshchagin ${ }^{1}$ (D), Ekaterina Pukhova ${ }^{1}$ (D), Margarita Khokhlova ${ }^{2}$ (iD) \\ ${ }^{1}$ Moscow Polytechnic University, Faculty of Information Technology, \\ Infocognitive Technologies, Moscow, Russia \\ ${ }^{2}$ LIRIS, Lyon Area, France
}

\begin{abstract}
Protection of goods and packaging from counterfeiting and copying, tracking their movement requires improvement of existing labeling and security methods and the development of new ones. Making changes to the image at the prepress stage is the cheapest and easiest way of protection compared to using special printing techniques, special substrates, and inks or additional tags such as RFID.

In the article, we suggest a new method to create security printed features, identify them in prints, and confirm the authenticity of the image. The method uses a combination of regular (AM) and stochastic (FM) screening in one image. There are two ways of separating images for AM and FM screening. First is to choose several random intervals in shadows of image tonal distribution and in accordance with values in these intervals original image is separated into two. The second is to separate by structure, for example, use FM screening on edges or textures. We tried Canny edge detector and local binary patterns. By using random values as the parameters, it is possible to generate unique print runs or even individual prints using digital printing. And large variability in the areas of separation gives reason to consider that the suggested method is reliable.

Fourier analysis in the suggested method allows not only to detect the presence of security printed features but also to confirm the authenticity of the image on a print. Authentication is implemented by obtaining a digital image of the print by scanning or photographing and comparing the spectral composition of the original image and the digital image of the print.

An expert survey showed that after our method presence of a combination of AM and FM screening in images on prints is barely visible. As a result, this method can be used to protect packaging labels with images from copying.
\end{abstract}

Key words: Fourier analysis, image processing, screening, halftone, security printing

\section{SECURITY PRINTING}

Today, the problem of protecting consumer goods from falsification is urgent. The simplest method of protection is to introduce protective elements into the product packaging, since packaging is the main element that identifies the product.

Most often, there is an image on the package that performs several functions at once: informing about the product and its purpose, attracts buyer, and indicates that it belongs to a particular brand. The image on the package is applied by printing, which implies the use of a complex multi-stage process. The complexity of the process allows us to use different methods to create security printed features in image. This can be the use of special methods of printing, materials, inks, or image processing methods that allow us to enter hidden information in addition to the original design. (Maresin, 2012)

It is possible to introduce protective elements into the package using one method or combine different ones (Mihalenko and Andreev, 2017). But it is necessary to consider that the cost of production of the package must have minimal impact on the cost of the final product, so if one of the methods is used that require additional costs or operations on the material stage of the process, then it is better to combine it with the protections implemented at the prepress stage (digital) when the image and the layout of the package is not yet put on media. Such methods require almost no additional costs.

To create secure packaging, security methods are needed at the digital stage that do not change the product design, for example, the introduction of hidden information - a marker (Mihalenko and Andreev, 2017). It is most advisable to insert such a marker into the image itself, which is part of the design, since most likely the packaging contains widespread open security methods, such as bar codes. The presence of a hidden marker is not obvious to the consumer, which makes this protection focused on regulatory authorities that use special means to identify such a marker. 
There are methods specially developed for the printing industry that allow introducing a hidden marker into an image at the digital stage: removing a tonal range, introducing a hardware-readable label in a color image, but these methods have restrictions on the area of introduction and are not suitable for all images and designs. (Andreev et al, 2014; Andreev et al, 2015)

Methods of introducing a hidden marker into a digital image are also widely used in steganography, but most of them are not resistant to transformations during printing, since they change the pixel structure of the image, which is converted during printing to raster.

The rasterization process affects the structure, gradation, and color of the image. There are two main rasterization methods - amplitude-modulated (AM) regular and frequency-modulated (FM) irregular. Each of these methods has its advantages and disadvantages, for example, regular and irregular structures reproduce the image tones and structure differently and have different resistance to moire formation (Gurjanova and Andreev, 2015). Currently there are algorithms and software that allows you to combine different parameters of the structure in a single image, such algorithms are aimed at improving tone reproduction or increasing sharpness of the graphical element edges (Samworth, 1997a; Samworth, 1997b; Shigeta, 2004).

Based on a combination of structures with different parameters, there are also methods for protecting images. The parameters that can be changed are frequency, dot shape, angle, and regularity (AM/FM). The area for introducing hidden information is selected by segmenting the image by spatial coordinates depending on the location of the composition elements in the image or design, which makes it easy to calculate the principle of introducing security tags. Identification of authenticity and detection of hidden information in such methods is carried out using templates with a regular structure that interacts with the structure of the print (Chugunov and Konstantinov, 2016; Zaicevskij, 2002).

\section{METHOD OF CREATING SECURITY PRINTED FEATURES}

A new method of introducing a hidden marker into the protected image was developed based on a combination of raster structures with different parameters that is not tied to the elements of the composition and does not require additional elements to be introduced into the design while being resistant to the process of tone value increase during printing reproduction.

The General algorithm of the method of introducing protection is that the image is divided into two according to a certain principle, then each of them is rasterized by the corresponding structure and then they are combined again (Figure 1). During the rasterization process, it is necessary to consider the difference in tone value increase for AM and FM structures and it can be done based on method described in following article (Pukhova, 2019).

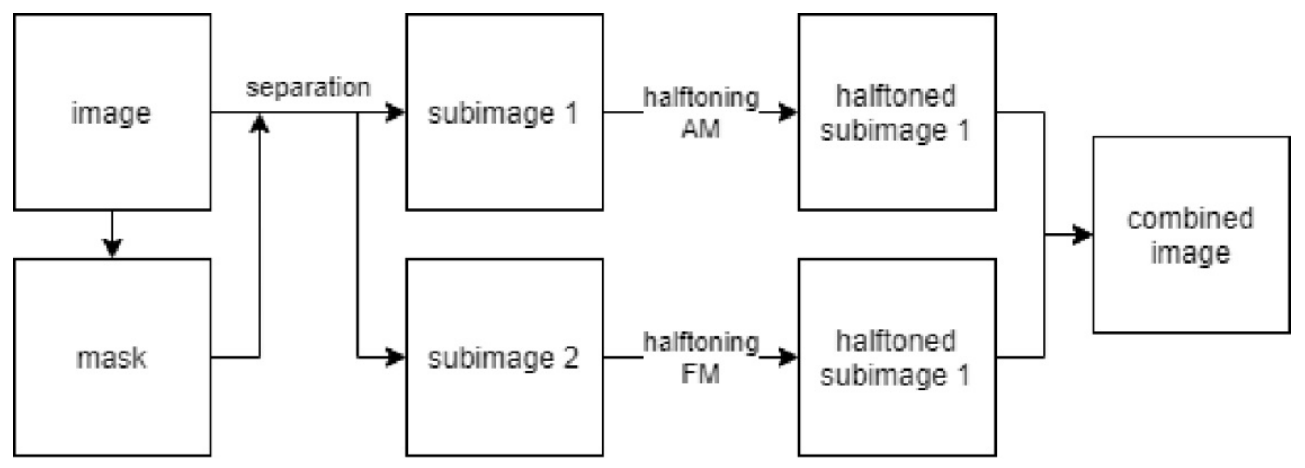

Figure 1: Flowchart for the suggested method of introducing security features

Two principles of image segmentation were chosen, which are difficult for a potential falsifier to calculate or copy: by tone value and by structure.

The degree of protection is achieved by the fact that for classical printing methods, the image can be changed from one print run to another, and then you can detect a discrepancy between the image and the period of its use, if the structure is completely copied. In the case of digital printing, it is possible to create each print unique, and if one image is repeatedly encountered, it can be concluded that there is a counterfeit. 


\subsection{Image separation by tone value}

The possibility of combining AM and FM structures when printing one image with the selection of the area for introducing FM structures in the selected tone range is considered. It is based on the method of removing the tone range (Andreev et al, 2015). The disadvantage of this method of protection is that the removed tone range is more than 40 levels of gradation, which in turn can lead to the effect of posterization in some parts of the images. Therefore, a method was proposed in which the removed tone range is replaced with a structure with changed parameters. Let us consider replacing it with an FM structure, while most of the image will be formed by AM structure.

To introduce the FM structure, a section of the tone range corresponding to the shadow area is selected, since an irregular structure has advantages over a regular one in shadows and at the borders of details (Daniel and Gonzalo, 2011). The introduction interval corresponds to 30\% of the total tone range of the image. Setting this range is determined by the zone theory, and in accordance with it, deep shadows and areas with a pronounced texture fall into the selected section of the tone range (Reinhard et al, 2002). If the range of image lightness [0:255], then the range of introduction of FM structure is [0:80], which corresponds to the size of the raster dots from $100 \%$ to $70 \%$.

When combining the FM structure with the AM, the difference in tone amplification of the two structures must be considered. The tone amplification effect is most pronounced for FM structures since with such structures a smaller point is formed. (Pukhova, 2019)

It is proposed to extract a subimage based on the image intensity histogram. We select image regions using the following scheme: The user defines the approximate desired percent of the image to be extracted (i.e, a percent of all the image pixels). Our recommendation is $30 \%$. Then a subimage with width of $\mathrm{N}$ bins is randomly selected and extracted from the histogram (Figure 2). The process in repeated until the number of pixels from the original grayscale image selected is equal (or bigger) than the percent specified by the user.

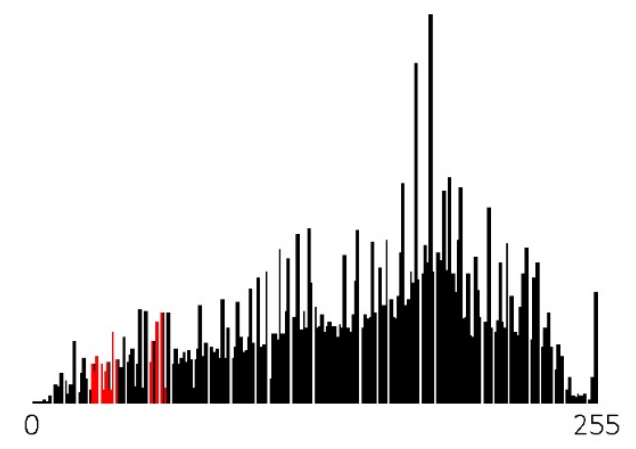

Figure 2: Example of resulting histogram (black) with selected regions (red) for extraction

Result of this stage is two subimages that will be halftoned differently (Figure 3). First subimage is used to introduce parameters that are variable relative to the second subimage, for example, it can be used for halftoning using a frequency-modulated raster structure. Also, several parameters can be replaced at once if it is necessary.
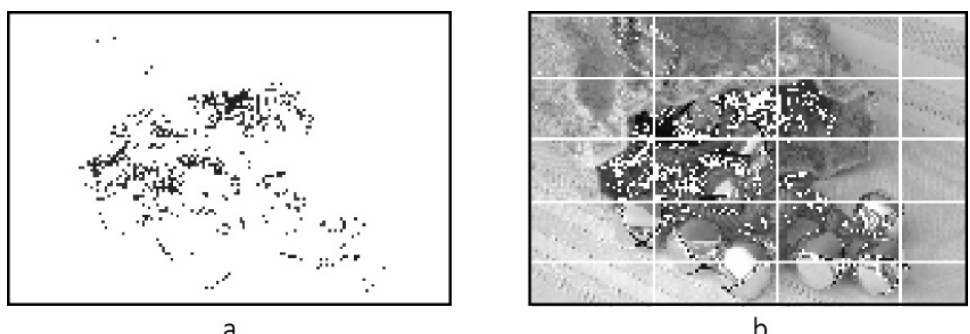

Figure 3: Subimages extracted from original image based on histogram: $a$-from red region (for FM structure), $b$-black region (for AM structure) 
Thus, before printing, two subimages are combined and this way image with security feature is generated. Exampled of combined image is shown in Figure 4.

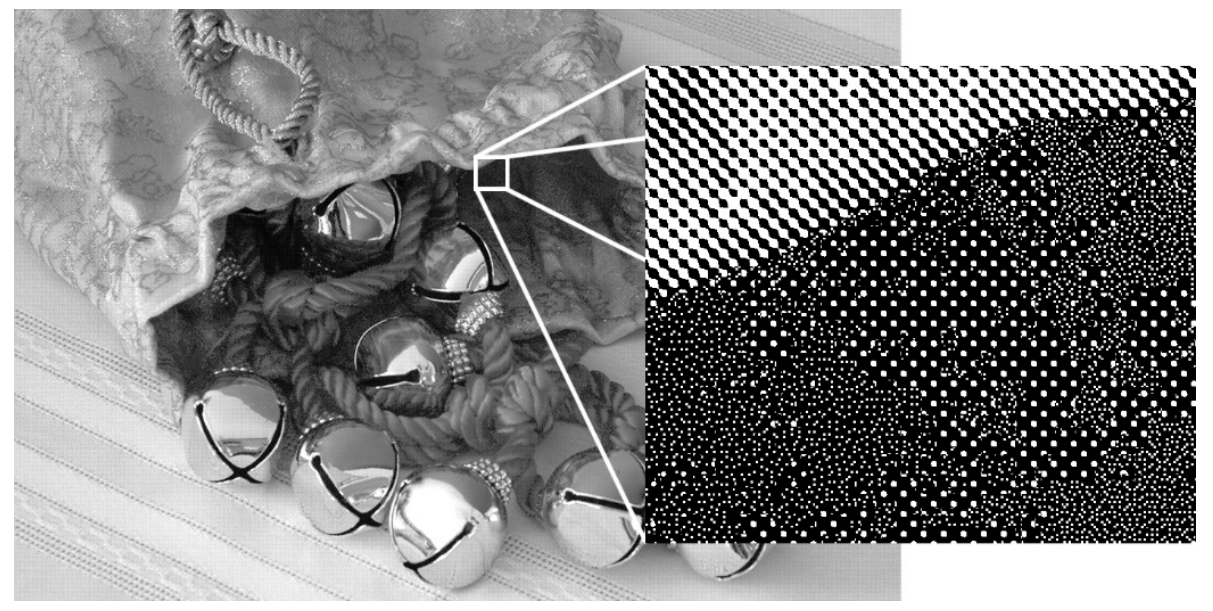

Figure 4: An example of combined image with magnified part that shows two structures

The proposed algorithm works for natural images, where the histogram has values in all the spectra, which are evenly distributed.

\subsection{Image separation by structure}

Another principle of introducing the FM structure into the image is by structure. In this work we used the Canny edge detector to extract edges from original image and Local Binary Patterns to extract corner-like regions.

For this specific image parameters for Canny edge detector were following:

- Standard deviation of the Gaussian filter - 2,

- Lower bound for hysteresis thresholding - 10,

- Upper bound for hysteresis thresholding -90,

and for Local Binary Patterns:

- Number of circularly symmetric neighbor - 24,

- Radius of circle -3,

- Method - uniform.

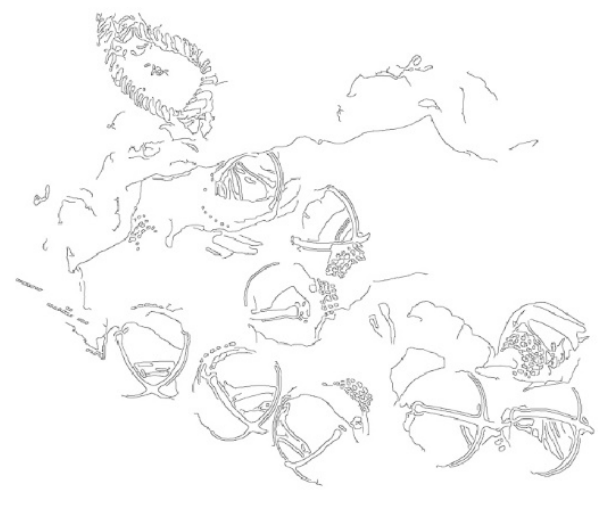

a

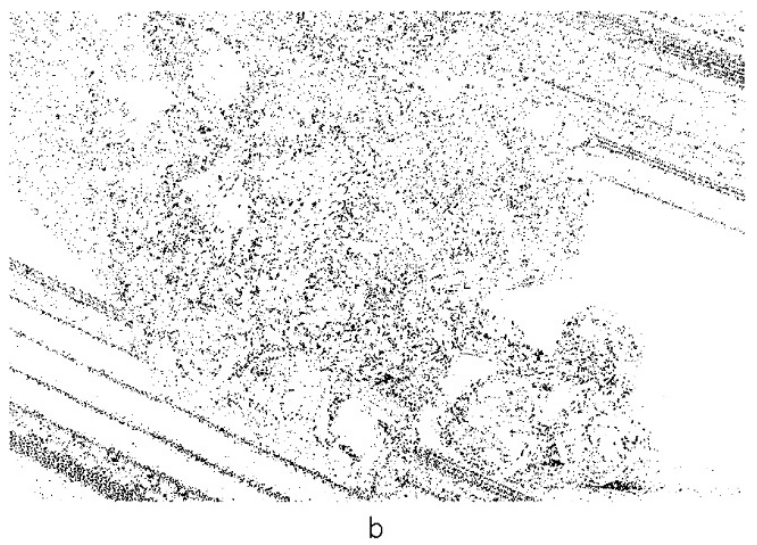

b

Figure 5: Examples of identifying areas by structure with: $a$ - Canny edge detector, $b$ - Local Binary Patterns with corner-like regions of the image 
By using this algorithm, we create masks to separate original image into two subimages, which will be halftoned with FM and AM structures accordingly and before printing combined creating an image with security features.

By making small changes in parameters of algorithms we can generate unique set of secured images.

\section{VISIBILITY OF SECURITY PRINTED FEATURES}

To check the visibility of security features in prints that were made with suggested method, an expert survey was conducted. Twelve experts were shown three images, which were printed without security features (Group 1), and with security features introduced by three ways (Group 2). The experts were asked the question "Do you notice any difference between images in group 1 and 2?" And the following answers were offered: "There is no difference", "The difference is hardly noticeable", "The images are clearly different". The results of the expert survey are shown in Table 1 - numbers are sum of given answers for each separation.

Table 1: Results of an expert survey on the visibility of security features in prints that were made with suggested method.

\begin{tabular}{|l|r|r|r|}
\hline & «no difference» & "hardly noticeable» & "clearly different» \\
\hline Separation by tone value & 27 & 8 & 1 \\
\hline $\begin{array}{l}\text { Separation by structure: } \\
\text { Canny edge detector }\end{array}$ & 22 & 12 & 2 \\
\hline $\begin{array}{l}\text { Separation by structure: } \\
\text { Local binary templates }\end{array}$ & 11 & 16 & 9 \\
\hline
\end{tabular}

According to the survey results, it can be concluded that the developed protection method has no visibility or sometimes hardly noticeable changes in image except separation with Local binary templates. Separation with Local binary templates require further research in finding optimal parameters. But at the same time, the presence of the introduced protective method can be detected using the Fourier analysis of the images and the authentication of the print can be performed when comparing the spectrum of the original image and the scanned one.

\section{SECURITY PRINTED FEATURES DETECTION AND IMAGE AUTHENTICATION}

To detect if printed image has suggested security features and authenticate the print, Fourier analysis is required. First step is to scan or photograph printed image. Then divide image back into two subimages using original masks and carry out spectral analysis. By the form of the spectrum we can detect the presence of AM or FM structures in the subimages.

If AM structure is present in the area with FM structure, then this will be noticeable in the image spectrum and vice versa, which allows us to conclude that the image is not genuine (Figure 6). For example, in the Figure $6(\mathrm{~d})$ presence of AM structure is identified by periodic peaks in spectrum, which are not expected in subimage that were halftoned with FM structure.

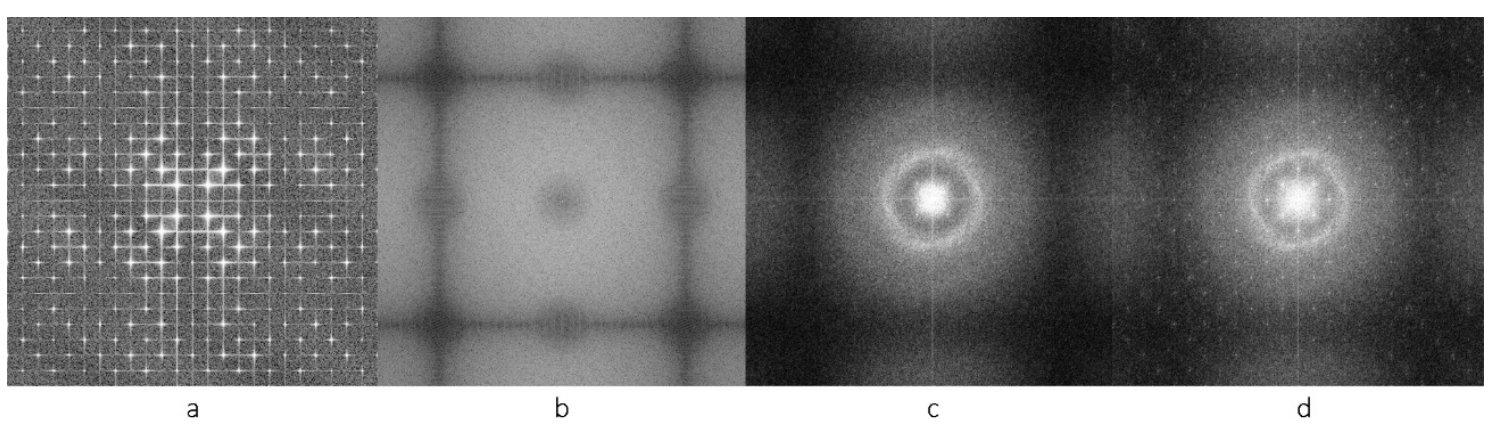

Figure 6: Image spectrum after Fourie transformation: a -AM structure, $b$-FM structure, c - FM structure of scanned image, $d$-FM structure of scanned image, where AM structure is present 
Also, it is possible to create database of original images, masks, and timestamps of generated images with suggested method and then use it to check if printed image authentic in case if someone copied the algorithm. It can be achieved by comparing the spectral composition of the original image and the digital image of the print.

\section{CONCLUSIONS}

As a result, a new method of protecting goods from counterfeiting and copying by introducing security features into images on labels and packaging was suggested. Method based on by combining AM and FM structures in one image. According to the results of an expert survey, changes in the images are visually hardly noticeable, except separation by Local binary templates with tested parameters. Fourier analysis of images allows to establish the authenticity of the examined prints.

Also, this method can be considered for the introduction of an additional dye corresponding in color to one of the colors of the triad, but different in that it has the property of fluorescence.

\section{REFERENCES}

[1] Andreev, Y. S., Pukhova, E. A., Tkacheva, M. V.: "Algorithm for introducing hidden hardware readable tags into a color image", Proceedings of higher educational institutions. Problems of printing and publishing 4, 3-8, 2014

[2] Andreev, Y. S., Pukhova, E. A., Yakhina, A. V.: "Application of the tonal range extraction method to protect information from unauthorized use", Proceedings of higher educational institutions. Problems of printing and publishing 4, 98-104, 2015.

[3] Chugunov, A. A., Konstantinov, V. A.: RU 2587 433, “Latent image acquisition method”, Полиграфзащита, 2016.

[4] Daniel, L., Gonzalo R.: 'Modern digital halftoning", (CRC Press, Boca Raton FL, 2011.), page 664.

[5] Gurjanova, O. A., Andreev, Y. S.: "A method for evaluating the fluctuation characteristics of halftone structures using the statistical parameters of the histogram", Proceedings of higher educational institutions. Problems of printing and publishing 3, 78-87, 2015.

[6] Maresin, V. M.: "Secured printing: reference book", (ФЛИНТА, Moscow, 2012.), page 640.

[7] Mihalenko, A. V., Andreev, Y. S.: "Rational classification of security technologies as the basis for designing a method for protecting printed products", Proceedings of higher educational institutions. Problems of printing and publishing 1, 24-29, 2017.

[8] Pukhova, E.: "Application of histogram method for valuation and compensation tone value changes, combining am-fm screens at press sheet", Proceedings of 3rd International Printing Technologies Symposium, (Printing Industry Education Foundation: Istanbul, Turkey, 2019), pages 119-124.

[9] Reinhard, E., Stark, M., Shirley, P., Ferwerda, J.: "Photographic tone reproduction for digital images", Proceedings of the 29th annual conference on Computer graphics and interactive techniques SIGGRAPH '02, (Association for Computing Machinery: San Antonio TX, USA, 2002), pages 267-276.

[10] Samworth, M.: US5892588A, "Digital halftoning combining dot size modulation screen with dot frequency modulation screen within a single image", PROFESSIONAL SOFTWARE TECHNOLOGIES Inc, 1997a.

[11] Samworth, M.: US6118935A, "Digital halftoning combining multiple screens within a single image", $1997 b$.

[12] Shigeta, K.: US20050155502A1, "Gravure printing method and gravure printed item", Think Laboratory Co Ltd, 2004.

[13] Zaicevskij, A.: LT-4922, "Method of technical protection of printed matter from falsification", 2002.

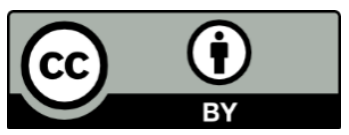

(C) 2020 Authors. Published by the University of Novi Sad, Faculty of Technical Sciences, Department of Graphic Engineering and Design. This article is an open access article distributed under the terms and conditions of the Creative Commons Attribution license 3.0 Serbia (http://creativecommons.org/licenses/by/3.0/rs/). 